\title{
Simulation and Analysis of Telescopic Photonic Beam Combiner for Stellar Interferometry in Labview
}

\author{
Simarpreet Kaur, Mohit Srivastava, Kamaljit Singh Bhatia
}

\begin{abstract}
The optical methods are the sensitive and subtle techniques of conducting optical investigations without any physical contact. They are both accurate and fast at the same time which provides an ease of having multiple observations. The need of optical interferometry has been increased at a very quick pace due to its high range of applications, from surface testing to locating of extra-solar planets in the universe. Optical Interferometry is a process or technique of combining light from various telescopes for calculating the angular resolution. The technique of optical interferometry helps astronomers for achieving a high angular resolution, that is not possible with the conventional telescopes. Optical approaches are the best approaches for non-contact evaluations, and these are accurate and fast. Due to optical nature of the interferometry, this process is used in various fields, and today it has become one of the important areas of research. Integrated Optics (IO) beam combiners are an efficient and compact technology to combine light interferometrically collected by multiple telescopes. IO beam combiner is based on the modal filtering properties of waveguides or fibers, and it improves thermo-mechanical stability, because of the characteristic rigidity of $I O$ component substrate. Other advantages of this technology are miniaturization and non-existence of alignment needs. Several IO beam combiners are being proposed and tested nowadays. The purpose of this paper is to investigate different techniques used to develop interferometric instrument from previous researches and to simulate the technique suggested by Ermann in Labview.
\end{abstract}

Index Terms: Beam Combiner, Condition Number, Integrated Optics Optical Interferometry, Stellar Interferometer, and Visibility To Pixel Matrix.

\section{INTRODUCTION}

Interferometry is a technique in which electromagnetic waves are superimposed on each other in order to create interference for extraction of useful information. Interferometry is a widely used technique which is used in astronomy, engineering and various other applications. Interferometry is mostly used in optical engineering which includes uses in fiber optics, optical metrology, spectroscopy, remote sensing, optometry, etc. The devices which are used for optical interferometry are optical interferometers which are used in science and engineering to measure refractive index, surface irregularities, and minute displacements [2].

Revised Manuscript Received on July 02, 2019

Simarpreet Kaur, kamaljeet Singh, IKG PTU Jalandhar, Punjab, India. Dr. Mohit Srivastava, Chandigarh Group of Colleges, Landran, Punjab, India.

The mechanism of working of an interferometer is that the light traveling from a source is split into two beams that travel into two optically different paths which are combined again to produce interference. The resulting interference fringes provide the information about the difference in the path lengths of two optical paths travelled by the splitting beam. Interferometers can be used to measure the lengths and shapes of optical components with precision up to nano-meters [5].

\section{A. Optical Interferometry}

In astronomy, optical interferometry is used to associate signals from two telescopes to get measurements with an advanced resolution which could not be obtained with any telescope separately. The technique is the source for astronomical interferometer arrays, which can take dimensions of minute astronomical objects when the telescopes are spread over a vast area. If a huge number of telescopes are taken for measurements, then an image can be formed, which has a resolution like a single telescope with the combined spread diameter of the telescopes. An interferometer is responsible for dividing the beam into several beams, and these beams travel by unequal paths, and their intensities which are added, reunited or subtracted, will interfere with each other. This interference looks like the patterns of dark and light bands which are known as interference fringes. These interference fringes are used for driving precise measurements about the thickness, to study lines of the spectrum and measuring refractive indices of materials that are transparent. Astronomers utilize interferometers for measuring star's distance and diameter [5].

\section{B. Principles of Optical Interferometry}

Following are the principles of optical interferometry:

Two-slit experiment of Young: This experiment states that when a monochrome light from a source at a distance, impinges on the two slits, the illumination pattern is estimated on the screen and observance of the fringe pattern is done. This model can be realized using interferometer in which light is received from two telescopes, and then these lights are combined using beam combination facility for the interference. The interference occurs due to the wavelength nature, in which, each slit propagates to the screen having different path lengths and ultimately these lights interfere at different points of the screen. Therefore, constructive interference condition can be defined as the intensity distribution's fringe spatial frequency proportional to 
the slit separation that is projected on the baseline $b$ and in units of observance of wavelength $\lambda[5]$.

Angular Resolution: This is defined as the capability of discerning the two components of a binary star system. Angular resolution is used for gauging the spatial resolution of an instrument. An interferometer resolves the equal brightness interferometry if the contrast of the fringe goes up to zero. This occurs when the angular separation becomes $\lambda / 2 b$, where $b$ refers to baseline [5].

Complex Visibility: The quantitative interpretation of the fringe patterns that are observed by interferometer can be made, and this is called visibility [5]. The visibility for the two-slit interferometer is given below:

$V=$ fringe amplitude/average intensity

The visibility is exactly proportional to the fringe amplitude and average intensity given by (1). Phase of the fringe pattern is equal to the Fourier phase of the same spatial frequency component. That is why; visibility is termed as complex visibility [5].

\section{Integrated Optics}

Integrated Optics can be defined as a technique which has its objective to construct integrated optical devices or various types of photonic integrated circuits or different types of planar integrated circuits which contain several optical components used together in order to perform certain complex functions [12].Various components which are used in integrated optics and its functions can be optical filters, amplifiers, photo-detectors, lasers, and modulators. The components used in integrated optics are made up on the surface of the materials which are crystalline in nature. Some common materials which are used in the fabrication of these components are silicon, $\mathrm{LiNbO} 3$, and silica, etc. and these components are connected with each other with the help of waveguides.

Integrated Optics is used for solving the problems of fiber optics. With fiber optics, it is easier to combine waves from two telescopes. But when more telescopes are involved, then combining lights from these telescopes is not an easy task, due to the fact that lights from different telescopes are split, combined many times and then lengths of the fibers are maintained and matched so that chromatic dispersion could be correct. However, this is difficult using the fiber optics. Therefore, integrated optics is used to resolve the problem of fiber optics [7]. Integrated optics can also be used for interferometric nulling. Interferometric nulling is identified as an emerging technology that can help in detection of the extra solar planets. In this approach, integrated optics is used which helps in combining an array of multiple telescopes in the nulling mode. Use of the integrated optics helps in measuring the angular transmission of telescope as in [12].

\section{Beam Combiners}

A beam combiner can be defined as an arrangement of beam splitters and mirrors [14]. The purpose of the beam splitter is to divide the light beam into two different paths for transmission and reflection. The beam splitters which are used in beam combiners are having a coating of a material that leads to the reflection of light which is monochromatic in nature and transmits the incident light by splitting it into the light of variable wavelengths. To have the minimum dispersion, the beam splitter is formed by the combination of three beam splitters. The coating which is used in beam splitters reduces the retardance of the incident light and avoids dispersion. Beam combiners are having a wide range of applications from laser beams to the combining of reflected light from planets in astronomy.

\section{Types of Beam Combiners}

The beam combiners are broadly categorized into two categories which are:

- Coherent Beam Combiners

- Spectral Beam Combiners

The goal of beam combining is to preserve the high quality of light beam and not only to generate the high output power. The combination of incoherent beams increases the dispersion for providing a solution to this problem. Beam combiners are used which separate the light beam first by splitting it, and then combines to preserve the quality of information.

Coherent Beam Combiners: These types of beam combiners are used in the combination of mutually coherent beams. One of the variants of the coherent beam combiners are the coherent polarized beam combiners. The other aspects are filled aperture and side by side combination of the light [7]. It can simply be defined as the monochromatic lights which are having similar optical frequencies combined using these types of combiners. The coherent beam combiners also work on the principle of combination of multiple frequencies when the emission spectra for all the frequencies are same. The coherent beam combiners can be used in the functioning process of ultra-short pulses [10].

Spectral Beam Combiners: Spectral beam combiners are also considered as incoherent beam combiners or the wavelength beam combiners because these types of combination techniques do not need mutual coherence of the light source. The spectral beam combiners work on the principle of using emitters with the use of non-overlapping emission spectra. Then every single beam is fed to the combiner, which is a wavelength sensitive beam combiner and uses components like, diffraction gratings, volume Bragg grating, dichroic mirrors or prisms [6]. 


\section{E. Photonic Beam Combiner}

A photonic beam combiner is responsible for combining the beams from different telescopes, so that, they can interfere with each other. The photonic beam combiner provides excellent performance in combining beams for astronomical interferometry. These combiners can provide small physical dimensions and thermo-mechanical stability that is suited for cryogenic assemblies. Photonic circuits are utilized in the existing instruments for combining four telescopes at the same time. Thus, these can help in enabling fast astronomical event's interferometric imaging. The photonic combiners simplify the integrated beam combiners' design, and thus it can be suited for various baselines and telescopes. There is no requirement of the alignment in the optical components of the photonic combiner, which makes it maintenance free and a stable combiner. The optical component used in these beam combiners helps to plug into different sources. It has several features such as; it is maintenance free and can combine multiple wavelengths [5].

\section{F. Discrete Beam Combiner}

A Discrete Beam Combiner uses a two-dimensional array of $M=N^{*} N$ coupled waveguides for combining the $N$ telescopes, as in Fig. 1. It works by injecting the light that is coming from the telescopes into the waveguides of the array. Then light propagation of one waveguide leaks to the nearest waveguides because of the evanescent coupling [18]. After this, the $\mathrm{N}$ input fields start interfering within the waveguides at given length. These input fields interfere at variable amplitude based on the length of the sample and observed waveguide. This interference helps in retrieving the mutual coherent characteristics of light from all pairs of telescopes.

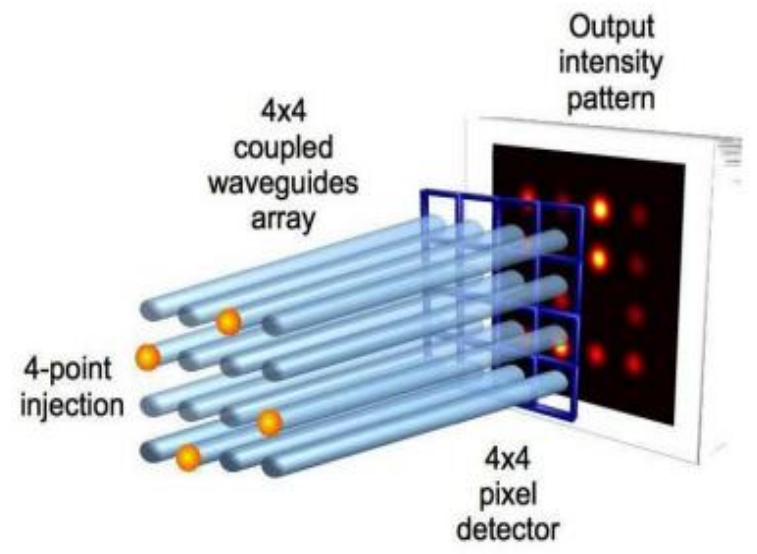

\section{Fig 1 : Discrete Beam Combiner [13]}

The operating principle of DBC is based on Van Cittert Zernike theorem, which relates the angular shape of a distant incoherent source to the Fourier transform of the complex spatial field correlation function (complex visibilities) of the emitted light [8].

\section{G. Stellar Interferometry}

Stellar Interferometry is the process of measuring the objects' angle, shape, and size. For example, close double stars can be measured. Michelson invented the instrument for the stellar interferometry that is known as the Michelson interferometer. Using this, light waves are received from two different telescopes and then that are combined so that interference can be produced. Stellar interferometry today has become the part of the toolbox of astronomers for observing unique capabilities of objects.

Stellar interferometry can be defined as a technique to observe the far away objects in space which is not possible by using a single powerful telescope used in optical interferometry [12]. Stars are the objects which are far away from the earth like the sun, and are much smaller in size compared to a one-dollar coin size.

These types of objects cannot be seen because of excessively small size and the diffraction of light that occurs in the path of light waves traveling across space. For solving this problem, an instrument was developed under stellar interferometry by Michelson and named as Michelson Interferometer which functions by combining lights to produce interferences which can be used to study the information of those far away objects [9].

\section{RELATED WORK}

J. D. Monnier, [5] focused on a new generation of large interferometers and also discussed basic principles of stellar interferometers and provides the new facilities under consideration. This study reviewed previous papers and found that very large telescope interferometers affect high resolution in astrophysics. The plane wave becomes corrupted when passes through the atmosphere. The astronomy experiences a great progress with optical interferometers. Stellar astrophysics is better in the area of star and planet formation.

P. Kern, and F. Malbet, [11] focused on single mode beam combiner for interferometry. Integrated optics provides self-alignment, improved resolution, and stability to the system. Single mode is also used in multi-beam combination, and guided optics is used to provide the single mode behavior for an application which demands accuracy. This study also discussed the existing technologies and current developments related to guided optics.

K, Parraut, and J. P. Berger, [7] discussed the principles of integrated optics, requirements for interferometry and the corresponding solutions provided by integrated optics. A combination of telescopes of an interferometer provides the coherent beam for stellar interferometry. Single-mode fibers are used to solve the problem of beam transportation. Guided optic is used for stellar interferometry by combining coherent beam coming from several telescopes of an interferometer. The integrated optic technique is an accurate and robust concept, and instrumental thermal emission can be fully controlled.

M. Olivero, and M. Svalbard, [9] used the ultraviolet writing for fabrication of optical beam combiner for stellar interferometry. Direct 
UV writings do not require photolithography. The two y-splitters were connected to a broadband coupler. Ultraviolet writings reduce the transmission loss and polarization losses. Optical interferometers are good for astronomical research. The device provides low crosstalk between photometric and interferometric channels. UV writings formed better fabrication techniques in stellar interferometry due to performance level and prototyping ability.

P. Benech, and P. Kern, [10] proposed a new detection technique to get details of an input signal with flux level measurements. When the guiding star fails to achieve a good signal to noise ratio, then coherence mode is used. Stationary Wave Integrated Fourier Transform Spectrometer (SWIFTS) based combiner is used for single mode interferometry. The SWIFTS concept provides the function for interferometric instruments that includes phase modulation, junction, switches, and detection. The photonic spectrometer was used to improve the signal to noise ratio.

H. Hsiao, and K. A. Winick, [4] designed the two beams, three beams, astronomical combiner and integrated optic interferometry combiner for operation in L-band. Integrated optics can provide multiple beams on a single chip. The three beam combiner is same as an integrated optic combiner. These devices were used in titanium-in-diffused, $x$-cut lithium niobate substrates. An on-chip electro-optic fringe scanning was also presented in this research. The white light fringes were generated with the help of two-beam combiner integrated along on-chip Y-splitter. The study concluded that it was possible to scan approximately 6 fringes using an applied $350 \mathrm{~V}$ of voltage.

S. Minardi, and T. Pertsen, [18] introduces a scheme of multiple beam combination in a three-dimensional array of coupled optical waveguide. The proposed method is based on the properties of two-dimensional arrays of coupled waveguides. Light propagation was same as conventional diffraction in an array of coupled waveguide. The array size is determined by coupling strength of waveguides. The study concluded that the proposed method could be used to determine the complex visibility of a target object observed by the interferometric array of three telescopes.

S. Minardi, and S. Nolte, [15] discussed astronomical interferometry which provides high-resolution information about astrophysical objectives. Three-dimensional photonic components have been used for multiple beams on a single chip. The work was performed in two phases. In the first phase, a combination of four telescopes is used with the monochromatic light source, and in the second phase, discrete beam combiner is used to manufacture a new sample of four telescope discrete beam combiner. The main aim was to characterize the component for broad wavelength band. MATLAB software was used for simulation.

C. Schmid [2] introduced stellar interferometry that is used in the field of Optical Quantum Information Science. The two techniques: Phase Referenced Imaging and Micro-Arc second
Astrometry have been used to change the polarization state of the incoming stellar light. The optical interferometers suffer from atmospheric as well as instrumental interferences. Integrated optic also provides filtering, improve stability, phase modulation and is used for wavelength conversion. Modeling is used to change the instrumental disturbances to the polarization state of stellar light. The research used different topologies for beam combination of two telescopes.

R. Errmann, and L. Labadie, [12] focused on integrated optic interferometry. Integrated optic components are used for four-beam nulling beam combiner. Four telescope beam combiner can generate a broad angular null. The authors measured the angular transmission of four telescopes with the motion of a star in the sky. One pair of telescopes with small distance is used to suppress the stellar light, and another pair is used for high resolution with large distance.

C. Enrico, et al. [3] analyzed numerically to study the effect of the thermally-induced refractive index which changes on the modes that propagate in a large area of double cladding fibers. Commercial software which is based on the finite element has been employed to calculate the refractive index and temperature over the cross-section of three PCF's (Photonic Crystal Fibers), exploited different strategies to enhance modal discrimination and obtained HOM (High Order Modes) suppression followed by a modal solver to calculate the guided modes. Yb-doped double-cladding PCF is the main factor for the high power fiber laser development.

Z. Houa, et al. [19] studied beam propagation formulation in an elliptical reflector in the nano-photonic integrated circuits (NPICs). A 2-D Gaussian beam mode is analyzed to formulate light beam transformation in an elliptical reflector. It was used to determine an elliptical reflector shape and placement, physical parameters of reflected beam such as waist size of the beam. The design of crossed waveguide which outperforms the conventional direct waveguide was crossed regarding the lowest-order mode of transmission efficiency and crosstalk. Curved reflector was the most convenient way to perform beam transformation function on optical lenses.

J. Tepper, et al. [6] analyzed full description of mid-IR IO two-telescope coupler and measured the performance levels for long-baseline interferometry. The research mainly focused on $\mathrm{L}$ and $\mathrm{M}$ astronomical bands exploitation. The experimental setup used the Michelson-interferometer to execute Fourier transform spectroscopy in order to measure the performance of broadband interferometer. Results presented the broadband interferometric measurement differences of $94.9 \%$ and $92.1 \%$ in $\mathrm{L}$ and $\mathrm{M}$ bands for unpolarized light. Moreover, 50\% of spectral integrated splitting ratio was observed with chromatic dependence on bandwidths. The phase variation across $\mathrm{L}$ and $\mathrm{M}$ bands was measured as $0.04 \mathrm{rad}$ and $0.07 \mathrm{rad}$, respectively, because of the coupler. Total throughput of $25.4 \%$ was observed for the coupler with Fresnel and injection losses. 


\section{SIMULATION OF DISCRETE BEAM COMBINER IN LABVIEW}

The intensities $I_{n}$ of the M waveguide modes at the end of the sample of waveguides can be related to the complex visibilities $\Gamma_{\mathrm{jk}}=\Gamma^{*}{ }_{\mathrm{kj}}=\left\langle A j A^{*}{ }_{\mathrm{k}}\right\rangle$ by a real valued, $\mathrm{M} \times \mathrm{N}^{2}$ elements matrix, called Visibility to Pixel Matrix (V2PM) as in [17] given in (2):

$I_{n}=\sum_{k=1}^{N^{2}}\{V 2 P M\}_{n, k} J_{k}$

Complex Visibilities $\Gamma$ are in the form of quadrature components $J_{i}$, which can be retreived from multiplication of $\left\{I_{n}\right\}$ with Pixel to Visibility Matrix $\{\mathrm{P} 2 \mathrm{VM}\}$, which is the pseudo-inverse of V2PM matrix. A practical method of evaluating the performance of a discrete beam combiner is to find its Condition Number $(\mathrm{CN})$ of V2PM matrix. $\mathrm{CN}$ is the ratio between the largest and smallest singular values of the V2PM matrix. The best configured waveguide configuratuion is the one with smallest condition number. The rectangular arrangement of waveguides $\mathrm{M}=\mathrm{N}^{2}$ in the range from 36 to 100 was searched in [13] and the condition number of 6 telescopes was calculated. That condition number has been plotted with various parameters in Labview.

\section{Condition Number Vs Number of Waveguides}

The below plot in Fig. 2 shows the variation of Condition Number with respect to the number of waveguides. It shows a clear monotonic decrease of the $\mathrm{CN}$ as the number of waveguides is increased. $\mathrm{CN}$ is decaying with increasing number of waveguides as in [13], but converges to 3.5 .

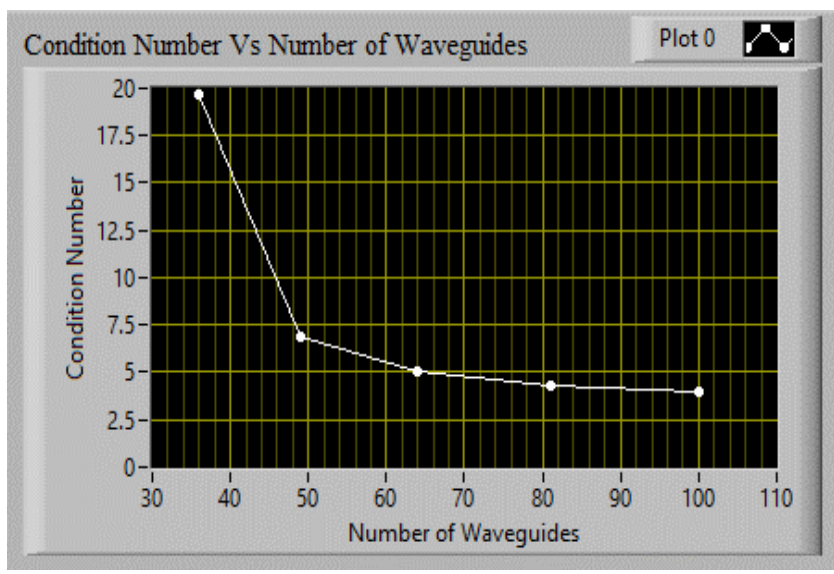

Fig 2: CN vs. Number of Waveguides

\section{Condition Number Vs Diagonal Coupling}

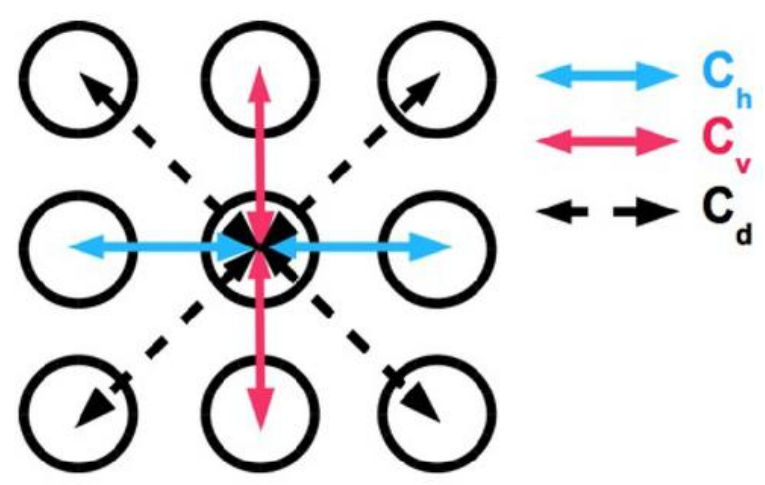

Fig 3: Coupling in DBC [13]

Fig. 4 shows the variation of $\mathrm{CN}$ with Diagonal Coupling. $\mathrm{C}_{\mathrm{d}}$ refers to diagonal coupling, and $\mathrm{C}_{\mathrm{h}}$ refers to horizontal coupling in waveguide array as shown in Fig. 3. This value needs to be inputted from the user side to specify the ratio of horizontal and diagonal coupling. At the end of the sample, the intensities of the $M$ waveguide modes can be related to the complex visibilities by a real valued, $M \times N^{2}$ element matrix, called Visibility to Pixel Matrix (V2PM). As mentioned in [13], "Tuning of the ratio $\mathrm{C}_{\mathrm{d}} / \mathrm{C}_{\mathrm{h}}$ above 0.1 can improve the conditioning of V2PM of DBC by $10-20 \%$."

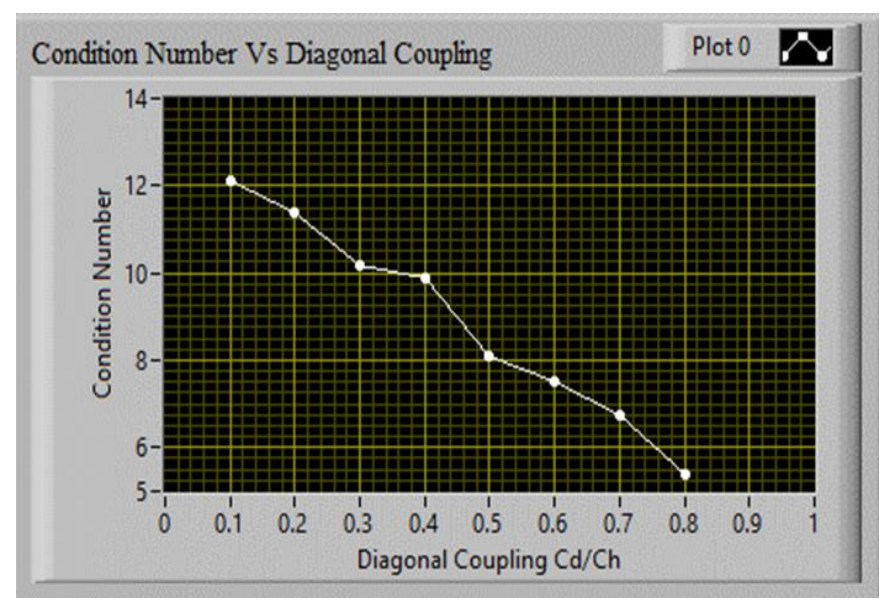

Fig 4: CN vs. Diagonal Coupling

\section{Condition Number Vs Bandwidth}

Waveguide coupling is strongly dependent on the wavelength of polychromatic light and it adversely affects the visibility and phase retrieval accuracy [16]. DBC can be used in spectro-interferometry mode and coherence retrieval method can be applied to small bandwidths. The bandwidth of the light source has been varied from a minimum of $1 \mathrm{~nm}$ to the maximum value as $60 \mathrm{~nm}$ as in Fig. $5 . \mathrm{CN}$ is varying constantly up to $40 \mathrm{~nm}$ and then increases sharply up to 60 nm.

The operation of the DBC can be extended to a total bandwidth of $50 \mathrm{~nm}$ centred at $650 \mathrm{~nm}$ by dispersing the output of the array. This

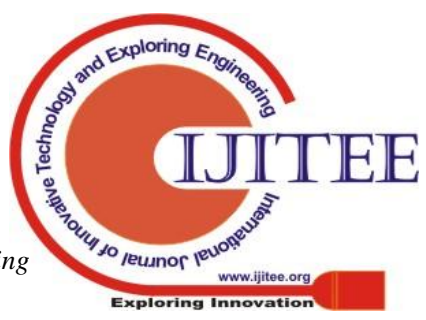


range can be extended by improving the design [1].

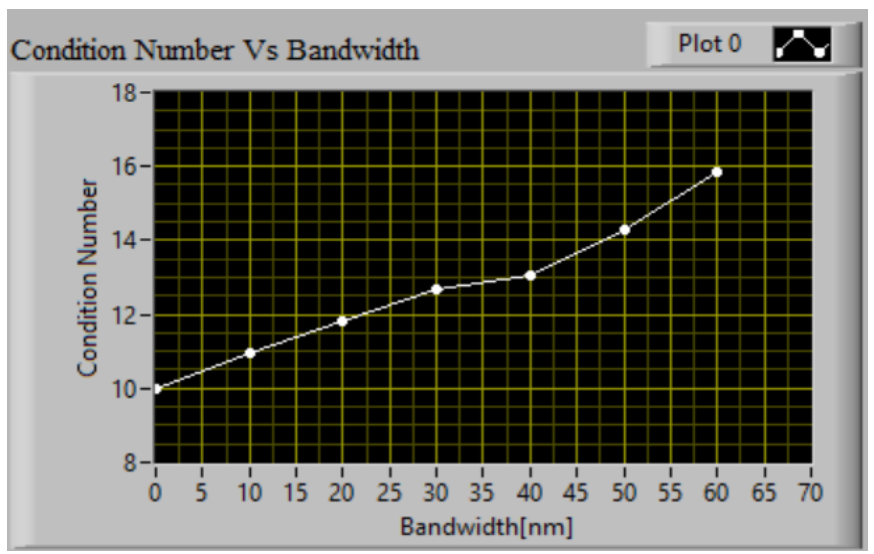

Fig 5: CN vs. Bandwidth

\section{1/SNR Vs Condition Number}

1/SNR vs. Condition Number variation is shown in Fig. 6. 1/SNR should increase with Condition Number, because CN represents the error or conditioning of the matrix. As the error increases, SNR decreases or 1/SNR increases. The dashed line at the bottom represents the theoretical photon-shot-noise limited visibility measurement error according to the given equation. Interestingly, this level corresponds to the theoretical per-baseline visibility error of a pair-wise $\mathrm{N}=6$ telescopes combiner measuring an average number of detected photons per telescope equal to $\mathrm{I}_{0}=10^{5}$ [13].

$$
\frac{1}{S N R}=\sqrt{\frac{N-1}{2 I_{0}}}
$$

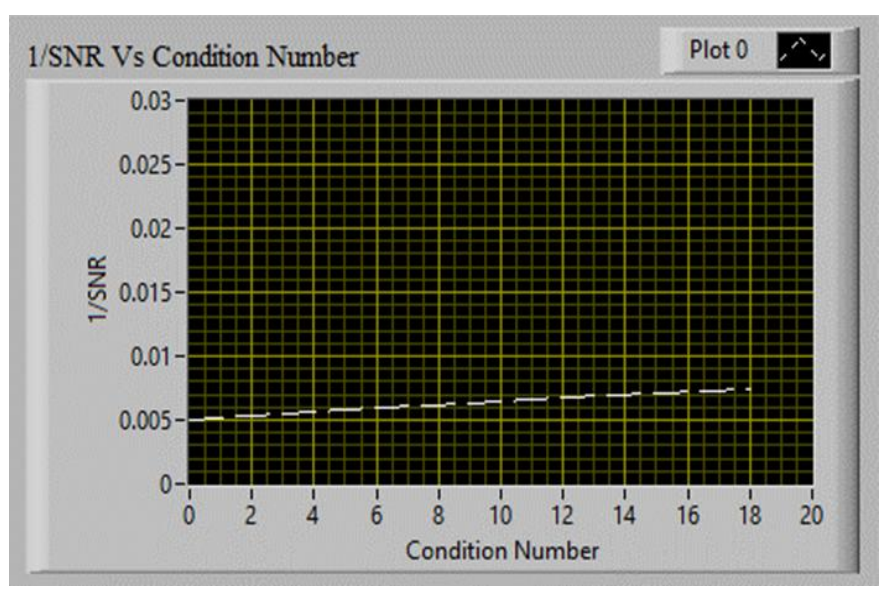

Fig. 6: 1/SNR vs. Condition Number

\section{CONCLUSION}

From the above review, it can be observed that the research done till now in the field of stellar interferometry, have shown experiments done with 4 or 6 telescopes using DBC architecture. For $8 * 8$ or $9 * 9$ DBC Arrays, only the input configurations were decided in the past researches. There is still a need to perform research to measure the SNR (Signal to Noise Ratio). In [13], it has been found that the performance of discrete beam combiner depends on the condition number of Visibility-to-Pixel-Matrix. The optimal waveguide configuration for the beam combiner of 6 telescopes has been evaluated by Discrete Beam Combiner (DBC), which is the regular array of straight, evanescently coupled waveguides. For 6 telescopes, it was found that performance increases monotonically with an increase in a number of waveguides. The gap lies in the fact that in [13], results for 8-telescope beam combiner are estimated by 6-telescope beam combiner results, but experiments have not been performed for the 8-9 - telescope beam combiner using the approach of IO discrete beam combiner featuring waveguide arrays. In the previous researches, there is already so much work done in $\mathrm{K}$, and $\mathrm{H}$ band. But limited work has been done by using L-band. So, there is the requirement to research more in this field to find the results using 8 or 9 telescopes and large DBC arrays in this band. In future, research can be performed to investigate an interferometric instrument using multiple telescopes with different combination schemes to obtain best-coupled waveguides for the larger arrays.

\section{REFERENCES}

1. A. Saviauk, S. Minardi, F. Dreisow, S. Nolte, and T. Pertsch, "3D-integrated Optics Component for Astronomical Spectro-Interferometry," in Appl. Opt. vol. 52, no. 19, 2013,4556.

2. C. Schmid, "Stellar Interferometric Beam Combiners in the Context of Linear Optics Networks," in Proceedings of SPIE 9146, 2014, 914632.

3. E. Coscelli, et al., " Analysis of the Modal Content into Large-Mode-Area Photonic Crystal Fibers under Heat Load," in IEEE Journal of Selected Topics in Quantum Electronics, vol. 22, no. 2, 2016 pp 323-330.

4. H. K.Hsiao and K.A.Winick, "An Integrated Infrared Optics Astronomical Beam Combiner for Stellar Interferometry at 3-4 um," in Optics Express, vol. 17, no. 21, 2009, pp 18489-18500.

5. J. D. Monnier, "Optical Interferometry in Astronomy," Journal of Lightwave Technology, vol. 66, 2003, pp 789-857.

6. J. Tepper, L. Labadie, R. Diener, S. Minardi, J. Pott, R. Thomson, \& S. Nolte, "Integrated Optics Prototype Beam Combiner for Long Baseline Interferometry in the L and M bands," in Astronomy \& Astrophysics 602, 2017, p A66.

7. K. Perraut, and J.P. Berger, "Planar Integrated Optics and Astronomical Interferometer," in Compt. Rend. Acad. Sci. Ser. IV Phys. Astrophys, vol. 2, no. 1, 2001, pp 111-124.

8. M. Born and E. Wolf, Principles of Optics (Cambridge U. Press, 1999), Ch. 10 .

9. M. Olivero and M. Svalbard, " Direct UV Written Integrated Optical Beam Combiner for Stellar Interferometry," in Journal of Lightwave Technology, vol. 25, no. 1, Jan. 2007, pp 367-371.

10. P. Benech and P. Kern, "On-chip Spectro-detection for Fully Integrated Coherent Beam Combiner," in Journal of Astrophysics, vol. 17, no. 3, 2009, pp 1976-87.

11. P. Kern and F. Malbet, "Single Mode Beam Combination for Interferometry," in Aerospace Conference JENAM , 2005, vol. 74, July. 2005, pp 5-6.

12. R. Errmann, and L. Labadie, (2015), "Interferometric Nulling of Four Channels with Integrated Optics, in Journal of Lightwave Technology, Applied Optics, vol 54, no. 24, 2015, pp 7449-54.

13. R. Errmann, and S. Minardi, "6 and 8 Telescope Discrete Beam Combiners," in Proceedings of SPIE, vol. 9907, Aug. 2016, pp 990733-1 - 33-8.

14. S. Minardi, et al, "Beam Combination Schemes and Technologies for the Planet Formation Imager," in Optical and Infrared Interferometry and Imaging V, SPIE, vol. 9907, 2016, pp 9907-57.

15. S. Minardi and S. Nolte, "Interferometric Nulling of Four Channels with Integrated Optics," in Applied Optics, vol. 54, no. 24, 2015, pp 7449-7454.

16. S. Minardi, A. Saviauk, F. Dreisow, S. Nolte, and T. 
Pertsch, “ 3D-Integrated Beam Combiner for Optical Spectro-Interferometry," in Optical and Infrared Interferometry IV, Proc. SPIE 9146, 2014, p. 91461D.

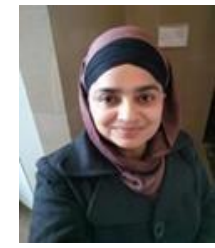

Simarpreet Kaur is a research scholar in Electronics and Communication Engineering Department at IKG Punjab Technical University, Jalandhar, Punjab, India. She received her B.Tech, and M.Tech in Electronics and Communication Engineering from PTU, Jalandhar in 2001 and 2008, respectively. She has about 43 publications in various International Journals, and participated in 11 National and 12 International conferences. Her current research interests are improving the performance of stellar interferometry systems and designing the best coupled waveguide discrete beam combiners.

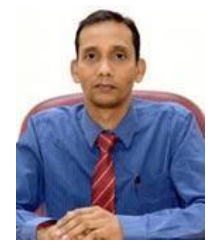

Dr. Mohit Srivastava is a Professor at the Department of Electronics and Communication Engineering and R\&D Dean at Chandigarh Engineering College, Landran, Mohali, Punjab, India. He received his B.Tech. in Electronics and Communication Engineering from Magadh University, Bodh Gaya, M.Tech. in Digital Electronics and Systems from K.N.I.T. Sultanpur and Ph.D. in Image processing \& Remote Sensing from Indian Institute of Technology Roorkee in 2000, 2008 and 2013 respectively. He has more than 15 years of work experience in various environments, including industry, as well as educational and research centers. $\mathrm{He}$ has completed two IEDC (DST) funded projects. His current research interests are digital image and speech processing, remote sensing and their applications in land cover mapping, as well as communication systems.

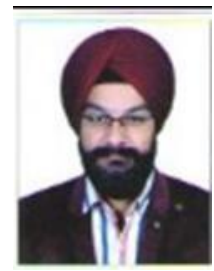

Dr. Kamaljit Singh Bhatia is an Associate Professor in Govind Ballabh Pant Institute of Engineering \& Technology, Pauri Garhwal, Uttrakhand, India. He has about 16 years of professional experience in teaching and research. He has more than 70 research publications in leadingNationalandInternationalJournals.Hehas authored four professional and engineering text books. He is the reviewer of many renowned International Journals and is life member of Optical Society of America (OSA), ISTE and Punjab Academy of Sciences. He is Technical Expert in IKG Punjab Technical University, Jalandhar, since 2010. His specializations are

17. S. Minardi, A. Saviauk, F. Dreisow, S. Nolte, and T. Pertsch, "Discrete beam combiners: 3D photonics for future interferometers," in Proceedings of Haute Provence Observatory Colloquium, vol. 23, no. 27, 2013, pp 121-129.

18. S. Minardi, and T. Pertseh, "Three-dimensional Photonic Combiners for Optical Astronomical Interferometry," in Proceedings of SPIE-The International Society for Optical Engineering, 7734, 2010.

19. Z. Houa, X. Lia, Y. Huang, and S.T. Hoa, "Physics of Elliptical Reflectors at Large Reflection and Divergence Angles I: Their design for nano-photonic Integrated Circuits and Application to low-loss low-crosstalk Waveguide Crossing," in Optical Communications, vol. 287, 2013, pp 96-105.

\section{AUTHORS PROFILE}

